\title{
ERROR BUDGET FOR A CALIBRATION DEMONSTRATION SYSTEM FOR THE REFLECTED SOLAR INSTRUMENT FOR THE CLIMATE ABSOLUTE RADIANCE AND REFRACTIVITY OBSERVATORY
}

\author{
Kurtis Thome, Joel McCorkel, Brendan McAndrew \\ NASA/GSFC
}

\begin{abstract}
A goal of the Climate Absolute Radiance and Refractivity Observatory (CLARREO) mission is to observe highaccuracy, long-term climate change trends over decadal time scales. The key to such a goal is to improving the accuracy of SI traceable absolute calibration across infrared and reflected solar wavelengths allowing climate change to be separated from the limit of natural variability. The advances required to reach on-orbit absolute accuracy to allow climate change observations to survive data gaps exist at NIST in the laboratory, but still need demonstration that the advances can move successfully from to NASA and/or instrument vendor capabilities for spaceborne instruments. The current work describes the radiometric calibration error budget for the Solar, Lunar for Absolute Reflectance Imaging Spectroradiometer (SOLARIS) which is the calibration demonstration system (CDS) for the reflected solar portion of CLARREO. The goal of the CDS is to allow the testing and evaluation of calibration approaches, alternate design and/or implementation approaches and components for the CLARREO mission. SOLARIS also provides a test-bed for detector technologies, non-linearity determination and uncertainties, and application of future technology developments and suggested spacecraft instrument design modifications. The resulting SI-traceable error budget for reflectance retrieval using solar irradiance as a reference and methods for laboratory-based, absolute calibration suitable for climatequality data collections is given. Key components in the error budget are geometry differences between the solar and earth views, knowledge of attenuator behavior when viewing the sun, and sensor behavior such as detector linearity and noise behavior. Methods for demonstrating this error budget are also presented.
\end{abstract}

Keywords: CLARREO, preflight calibration, radiometric calibration, SI-traceable

\section{INTRODUCTION}

The Climate Absolute Radiance and Refractivity Observatory (CLARREO) has the science objective of making highaccuracy, SI-traceable decadal change observations with the goal of allowing a better understanding of climate change predictions. Details of the mission and the science behind can be found in Wielicki et al. [1]. The efforts to define the CLARREO mission led to three key conclusions about measurement needs: 1) traceability to fundamental SI standards; 2) temporal and angular sampling to detect decadal climate change signals above natural variability; 3 ) sufficient information content to infer climate change in the key climate change variables of interest.

Converting the above three needs into a measurement program resulted in pairs of instruments on separate platforms in 90-degree, precessing orbits. The instrument suite receiver on each spacecraft included either a single emitted infrared spectrometer with one global navigation receiver for radio occultation or a single reflected solar instrument. The measurements were to be acquired for a period of three years minimum, with a five-year lifetime goal, enabling followon missions to extend the climate record over the decades needed to understand climate change. Accuracy requirements sufficient to observe climate change to within the uncertainty of the limit of natural variability were shown to be $0.1 \mathrm{~K}$ $(\mathrm{k}=3)$ for the infrared spectrum and $0.3 \%(\mathrm{k}=2)$ for the reflected solar spectrum [1].

*kurtis.thome@nasa.gov; phone 1301 614-6671

Imaging Spectrometry XVIII, edited by Pantazis Mouroulis, Thomas S. Pagano, Proc. of SPIE Vol. 8870, $887008 \cdot$ C 2013 SPIE · CCC code: 0277-786X/13/\$18 · doi: 10.1117/12.2024562 
The current work describes the error budget for the reflected solar (RS) instrument of CLARREO. The instrument will retrieve at-sensor reflectance over the 320 to $2300 \mathrm{~nm}$ spectral range with 500-m spatial resolution and 100-km swath. Reflectance is obtained from the ratio of measurements of the earth's surface to those while viewing the sun relying on a calibration approach that retrieves reflectance with uncertainties less than $0.3 \%$. Further details on the RS instrument can be found elsewhere $[1,2]$ as well as discussion of a preliminary error budget developed to understand the most challenging aspects of the CLARREO reflectance retrieval [2].

CLARREO, at the time of this paper, remains in an extended pre-phase A studying mission science trades, engineering trades, S.I. traceability risk reduction, and optimization of CLARREO science. A set of calibration demonstration systems (CDS) have been developed to minimize the risk related to the stringent accuracy requirements of CLARREO and demonstrate the feasibility of making measurements of the required accuracy.

The CDS for the reflected solar part of the spectrum, SOlar, Lunar for Absolute Reflectance Imaging Spectroradiometer (SOLARIS), was constructed to develop and check calibration protocols and methods, demonstrate the path to SI traceability (source and detector standards), and prove the ability to derive reflectance via a view of the sun and scene [3]. The test plan previously described for SOLARIS provides better understanding of the CLARREO error budget given here. The paper begins with a brief overview of the RS design and CLARREO on-orbit calibration approaches followed by discussion of CLARREO uncertainty sources. The uncertainties related to CLARREO and the approach to prelaunch calibration is described and an updated error budget is presented showing what is needed to achieve the CLARREO calibration level of $0.3 \%$.

\section{REFLECTED SOLAR (RS) SENSOR DESCRIPTION}

A preliminary design for the CLARREO RS has been given previously [2] and is summarized here for completeness. The sensor would operate from 320 to $2300 \mathrm{~nm}$ with 500-m ground-projected instantaneous-field-of-view (GIFOV) and a $100-\mathrm{km}$ swath width. Reflectance is retrieved through a ratio of earth-viewing data to solar-viewing measurements. The RS instrument accounts for the large difference in illumination levels while maintaining accurate retrieval of the reflectance traceable to SI standards at a level better than $0.3 \%$ in the mid-visible. Table 1 summarizes the driving instrument requirements for the RS sensor that would provide the data necessary to achieve a benchmark climate data set through CLARREO measurements as well as via intercalibration of other sensors allowing them to derive a benchmark.

The instrument approach is an Offner imaging spectrometer operating as a pushbroom scanner relying on heritage hardware to reduce sensor complexity. Two separate focal planes based on silicon and MgCdTe detectors are coupled with existing Indigo 9803640 x 512 pixel read-out integrated circuits (ROICs). Each focal plane is contained within its own spectrometer having a separate entrance aperture, grating, and focal plane that are nearly identical. Each camera includes front-mounted, rotatable attenuator wheels. The blue spectrometer covers 320-640 nm with a conventional, single blaze diffraction grating. The red spectrometer covers $600-2300 \mathrm{~nm}$ via a double blaze diffraction grating, coupled with an order-sorting filter. The overall mass of a single spectrometer is $20 \mathrm{~kg}$. Attenuator approaches include a single pinhole aperture, neutral density filters, a collection of pinhole apertures, or various combinations of the three.

\section{ON ORBIT CALIBRATION}

SI traceability is the cornerstone of the success of CLARREO's mission. The basis for the long-term SItraceability on orbit is the sun itself through the determination of reflectance. One reason for adopting a reflectance philosophy is it reduces the need for elaborate onboard calibration sources. Conversion of the reflectance

\begin{tabular}{|l|l|}
\hline Parameter & $\begin{array}{l}\text { Instrument Measurement } \\
\text { Requirement }\end{array}$ \\
\hline $\begin{array}{l}\text { Radiometric } \\
\text { Accuracy }\end{array}$ & $0.3 \%$ in reflectance of albedo \\
\hline Spectral Range & $320 \mathrm{~nm}$ to $2300 \mathrm{~nm}$ \\
\hline Spectral Resolution & $8 \mathrm{~nm}$ \\
\hline Spectral Sampling & $4 \mathrm{~nm}$ \\
\hline $\begin{array}{l}\text { Instantaneous Field } \\
\text { of View (IFOV) }\end{array}$ & $<0.5 \mathrm{~km} \times 0.5 \mathrm{~km}$ \\
\hline $\begin{array}{l}\text { Signal-to-Noise- } \\
\text { Ratio }\end{array}$ & $\begin{array}{l}\text { SNR }>33 \text { at } 0.3 \text { reflectance } \\
\text { for } 75 \text { degree solar zenith }\end{array}$ \\
\hline Swath Width & $<100 \mathrm{~km}$ \\
\hline $\begin{array}{l}\text { Polarization } \\
\text { Sensitivity }\end{array}$ & $\begin{array}{l}<0.50 \% \text { below } 1000 \mathrm{~nm} \text { and } \\
<0.75 \% \text { above } 1000 \mathrm{~nm}\end{array}$ \\
\hline
\end{tabular}

Table 1. Driving instrument requirements for RS instrument 
to an absolute radiance will rely on access to an appropriately accurate solar irradiance. The retrieval of the atCLARREO bi-directional reflectance distribution function (BRDF) at wavelength, $\lambda$ for detector $\mathrm{i}$ of the sensor can be given as

$$
B R D F_{\mathrm{i}, \lambda}^{\text {sensor }}=\frac{\mathrm{L}_{\mathrm{i}, \lambda}^{\text {earth }}}{\mathrm{E}_{\mathrm{solar}, \lambda} \cos \vartheta_{\text {solar }}}
$$

where $\theta_{\text {solar }}$ is the solar zenith angle incident at the top of the earth's atmosphere, $E_{i, \lambda}^{\text {solar }}$ is the total solar spectral irradiance at the earth-sun distance at the time of the measurements and $L_{i, \lambda}^{e a r t h}$ is the spectral radiance measured while viewing the earth. The solar irradiance can be written in terms of the sensor output while viewing the sun, $S_{i, \lambda}^{\text {solar }}$, and $\mathrm{R}_{\mathrm{i}, \lambda}$ which is the responsivity of $\mathrm{i}^{\text {th }}$ detector and wavelength, $\lambda$, as

$$
\mathrm{E}_{\text {solar }, \lambda}=\frac{\sum_{\mathrm{x}_{\text {solar }}^{\prime}} \sum_{\mathrm{y}_{\text {solar }}^{\prime}} \mathrm{S}_{\mathrm{i}, \lambda}^{\text {solar }}\left(\mathrm{x}_{\text {solar }}^{\prime}, \mathrm{y}_{\text {solar }}^{\prime}\right)}{\mathrm{R}_{\mathrm{i}, \lambda}^{\text {sensor }} \mathrm{T}_{\text {attenuator }} \mathrm{A}_{\text {attenuator }}}
$$

where $T_{\text {attenuator }}$ is the transmittance of the attenuator used to allow direct view of the solar disk, and $A_{\text {attenuator }}$ is the entrance pupil of the sensor when viewing the solar disk. The summation over $\mathrm{x}_{\text {solar }}$ and $\mathrm{y}_{\text {solar }}$ integrates the output from a single detector over the entire solar disk needed to measure the solar irradiance. The use of irradiance mitigates effects due to spatial heterogeneity of the solar disk.

In like fashion, the earth-view radiance $L_{i, \lambda}^{\text {earth }}$ can be written in terms of the responsivity as

$$
\mathrm{L}_{\mathrm{i}, \lambda}^{\text {earth }}=\frac{\mathrm{S}_{\mathrm{i}, \lambda}^{\text {earth }} \mathrm{A}_{\text {sensor }} \Omega_{\text {sensor }}}{\mathrm{R}_{\mathrm{i}, \lambda}^{\text {sensor }}}
$$

where $\mathrm{A}_{\text {sensor }}$ is the area of the entrance pupil of the sensor while viewing the earth, $\Omega_{\text {sensor }}$ is related to the field of view of the sensor, and $S_{i, \lambda}^{e a r t h}$ is the signal from the given detector at the specific wavelength while viewing the earth.

Substituting for the solar irradiance and earth-view radiance in (1) leads to

$$
B R D F_{\mathrm{i}, \lambda}^{\text {sensor }}=\frac{\mathrm{S}_{\mathrm{i}, \lambda}^{\text {earth }}}{\mathrm{R}_{\mathrm{i}, \lambda}^{\text {sensor }} \mathrm{A}_{\text {sensor }} \Omega_{\text {sensor }}} \frac{\mathrm{T}_{\text {attenuator }} \mathrm{A}_{\text {attenuator }} \mathrm{R}_{\mathrm{i}, \lambda}^{\text {sensor }}}{\cos \vartheta_{\text {solar }} \sum_{\mathrm{x}_{\text {solar }}} \sum_{\mathrm{y}_{\text {solar }}} \mathrm{S}_{\mathrm{i}, \lambda}^{\text {solar }}\left(\mathrm{x}_{\text {solar }}^{\prime}, \mathrm{y}_{\text {solar }}^{\prime}\right)}
$$

where it is assumed that any temporal changes in response between the solar and the earth views are minimal. In theory, one does not need an absolute radiometric calibration of the RS instrument in order to achieve an accurate determination of reflectance. What is required is knowledge of the attenuator transmittance and the physical geometry factors related to the optical system.

Ensuring SI-traceability and adequate accuracy still requires evaluation of sensor performance on orbit and this will rely on a combination of calibration, validation, and verification activities. Verification is defined here as any method that ensures the fidelity of the calibration methods either by demonstrating SI-traceability, ensuring that SI traceability is maintained, or tracking temporal degradation of calibration mechanisms. The verification approach is either by demonstration of collected and calibrated spectral data or by a combination of on-orbit test images combined with data analysis.

Validation evaluates data from the sensor relative to an independently-derived data set. One approach planned for validation of the RS on-orbit calibration will be comparisons to on-ground measurements propagated through the atmosphere to predict at-sensor radiance. Another will be comparisons to other sensors including airborne sensors. The main difficulty with validation for CLARREO RS will be ensuring that the validation data sets have sufficient radiometric quality to provide useful results.

Calibration of the RS instrument on orbit relies primarily on direct solar views including a set of attenuation filters to reduce the irradiance from the sun to a level that is consistent with the radiance expected from reflected energy from the Earth's surface. The calibration of the attenuating systems takes place during the prelaunch calibration efforts using NIST-developed techniques. 
Multiple solar and lunar collections are planned including several intensive solar imaging operations early in the mission intended to calibrate the entire focal plane of the RS. Comparison of the measured solar irradiance to other wellestablished values provides a validation of the RS sensor. Periodic solar collections allow evaluation of the sensor as a function of time. The solar collections are paired on a monthly basis with the lunar collections to verify the quality of the attenuating system.

\section{UNCERTAINTY SOURCES}

As mentioned, the system relies on a direct solar view as the primary calibration approach while on orbit. Examination of (4) shows three basic terms: 1) solar measurement, 2) earth measurement, and 3) spectral dependence. The spectral dependence refers to uncertainties in the knowledge of the spectral characteristics of the spectrometer such as spectral band shape (wavelength calibration artifacts) or caused by the interaction of the spectrometer's slit with the imaging spectrometer (line spread function artifacts).

Rewriting (4) to include error terms results in

$$
B R D F_{\mathrm{i}, \lambda}^{\text {sensor }}=\frac{\mathrm{S}_{\mathrm{i}, \lambda}^{\text {earth }}}{\mathrm{A}_{\text {sensor }} \Omega_{\text {sensor }}} \frac{\mathrm{T}_{\text {attenuator }} \mathrm{A}_{\text {attenuator }}}{\cos \vartheta_{\text {solar }} \sum_{\mathrm{x}_{\text {solar }}} \sum_{\mathrm{y}_{\mathrm{solar}}} \mathrm{S}_{\mathrm{i}, \lambda}^{\text {solar }}\left(\mathrm{x}_{\text {solar }}^{\prime}, \mathrm{y}_{\text {solar }}^{\prime}\right)} \frac{\mathrm{a}_{\text {sensor }}^{\text {straylight }} \omega_{\text {sensor }}^{\text {straylight }} \mathrm{a}_{\text {attenuator }}^{\text {straylight }}}{\mathrm{r}_{\mathrm{i} \text { fleld }}^{\text {flat }} \mathrm{r}_{\mathrm{i}, \lambda}^{\text {nonlinearity }} \mathrm{r}_{\mathrm{i}, \lambda}^{\text {plarization }}} \quad \text { Eq. } \mathbf{5}
$$

where lower case variables relate to their upper case counterparts and the superscript labels the source of the uncertainty for that variable. Stray light is considered as any light path that deviates from a perfect geometrical optics path including diffraction as well as spurious reflections. The detector responsivity, R, suffers uncertainties caused by the polarization of the optical system as well as non-linearity effects in the detectors. The "flat-field" term refers to the fact that full independent characterization of all detectors may not be feasible and a method for transferring the calibration of one detector to another may be needed.

The early error budget analysis identified the above uncertainty sources as the dominant terms to consider realizing, of course, that each of these terms has many contributing factors [3]. Early efforts on the CLARREO project led to identifying the best approaches to characterize a sensor relying on (4) to retrieve reflectance while maintaining high accuracy and SI-traceability over the lifetime of the mission [2]. Those efforts led to the basic calibration flow shown in Figure 1 (reproduced from [2]). The demonstration of SI traceability requires a clear demonstration of the accuracy of the ratio in (4) and then a transfer of that calibration to orbit and over the lifetime of the mission. SI-traceability is tied to the electric Watt during prelaunch testing. The radiance to irradiance ratio is assessed through absolute calibration of the sensor in irradiance mode and radiance mode and determination of geometric factors for conversion to reflectance.

The sensor model shown in Figure 1 can be thought of as the numerical abstraction of the physical instrument, encapsulating knowledge of both optical physics, and empirical results gained from laboratory analysis of the engineering model. Laboratory measurements made during the prelaunch phase using various calibration devices are compared with model predictions for agreement, and the identified disparities are used to guide model improvement. This is a continuous process that ultimately yields a sensor model ready for use after launch.

The end result of the prelaunch calibration is sufficient data to develop a full sensor model

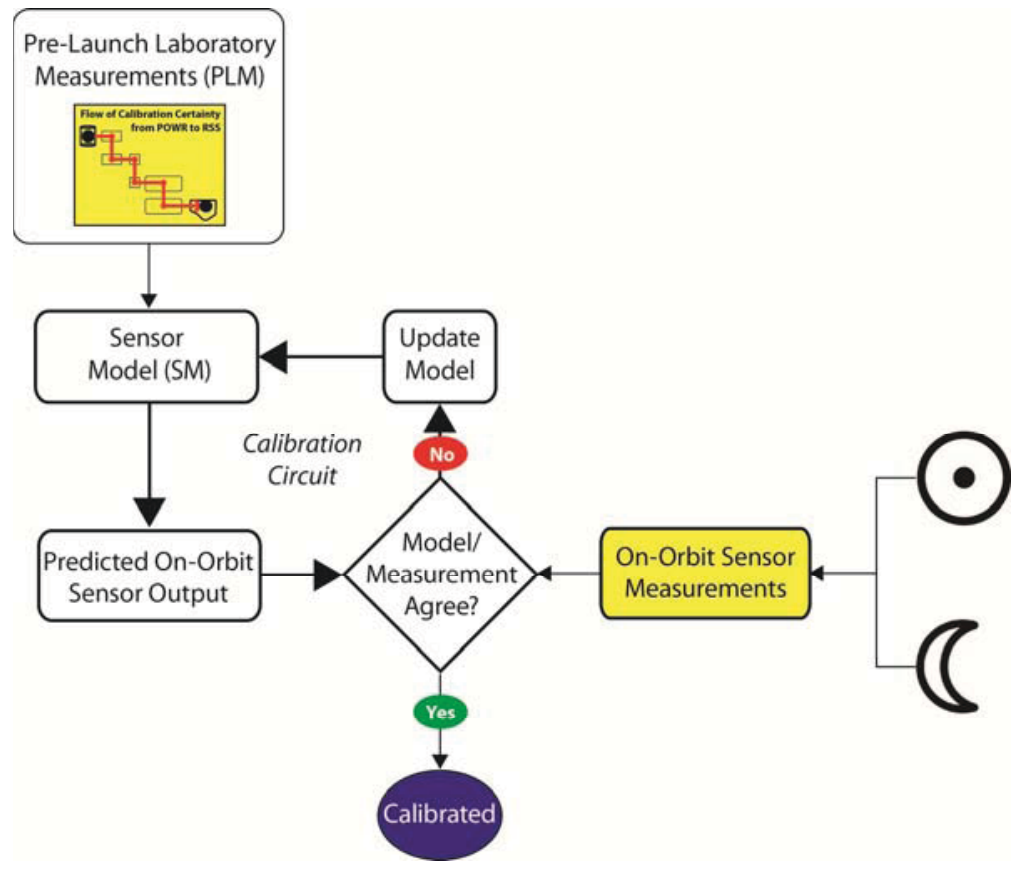

Figure 1. RS Instrument calibration process-flow diagram 
which can predict expected sources to be used for on-orbit calibration: sun, moon, and other planetary/stellar sources. Such an approach permits the on-orbit calibration to validate the prelaunch calibration. Agreement between prelaunch and on-orbit (as shown in Figure 1) means the system is calibrated, and by analogy traceable to the pre-launch SI measurements. Disagreement is inferred to mean that the sensor model requires improvement based on the on-orbit data, and would likely include an additional set of characterization measurements. Once the on-orbit traceability is established with a demonstrable error budget, the solar and lunar views provide information regarding temporal changes in the sensor. Thus, the key to the RS on orbit calibration is the prelaunch, SI-traceable calibration.

Calibration systems, such NIST's Spectral Irradiance and Radiance Responsivity Calibrations using Uniform Sources (SIRCUS) facility, provide a standard capable to understand stray light, spectral response, and polarization sensitivity at the level necessary for CLARREO [4]. The basis of SIRCUS is a well-understood tunable laser source that can be coupled to a fiber optic system providing both radiance and irradiance sources. The output of the source is determined via detector standards characterized against the Primary Optical Watt Radiometer (POWR). A transfer radiometer calibrated at NIST facilities to the electric Watt is coupled with a tunable laser system to calibrate the output of the sources used in the calibration of the RS instrument. The stated accuracy to calibrate a transfer radiometer in irradiance mode using POWR is $0.09 \%$ at $\mathrm{k}=3$. The transfer radiometer characterization of the output of a monochromatic laser source coupled to a spherical integrating source is $<0.2 \%$ for $\mathrm{k}=3$ [4].

\section{PRELAUNCH TEST PLAN}

The overall approach in Figure 1 has been used to develop a test plan for SOLARIS, the RS CDS, which is the basis for the calibration test plan for the CLARREO RS [3]. The main objectives to testing of SOLARIS are to:

1) Develop and evaluate calibration protocols leading to an SI-traceable calibration of SOLARIS

2) Develop a physically-based spectrometer model

3) Create a defensible error budget

The test plan relies strongly on the absolute calibration against SIRCUS. SOLARIS testing is ongoing at the time of the current work, but early results provide the updated error budget for CLARREO presented in the next section as part of addressing objective 3. The CLARREO tests are summarized here and further details can be found in [3].

Initial testing of SOLARIS took place at the component and subsystem level prior to assembly of the sensor. The key components characterized were optical elements including the slit and grating, the detector package, and attenuation and depolarizer elements. The next stage of testing worked to develop the laboratory source knowledge at $<1 \%$ absolute (all values $\mathrm{k}=2$ ) through calibration of the CXRs.

Emphasis of the laboratory testing is on the radiometric and spectral characterizations since the current state of the art of geometric and spatial calibration approaches are sufficient for CLARREO mission requirements, assuming that stray light, scattered light, and ghosting analysis are radiometric properties. The importance of stray light in the reflectance retrieval makes characterization and modeling of stray and scattered light crucial for SOLARIS and the field-based measurements of the sun and surface reflectance retrievals essential to demonstrate understanding of the error budgets.

The first round of testing is effectively complete at the time of the writing of this paper and the components have been included in the fully integrated instrument. Full evaluation of these data is still occurring and the results will be used as part of the error budget development and instrument model. A key advantage to SOLARIS is that it can be disassembled allowing the components to be reevaluated if it is determined that further data are needed to improve the instrument model or to understand and verify the error budgets. Ideally, the components are evaluated to a level at which the uncertainties in the characterization data do not detrimentally affect the instrument-level error budgets.

\section{ERROR BUDGET ANALYSIS}

Figure 3 shows the overall estimate of the at-CLARREO reflectance uncertainty in percent of reflectance (all error values in this work are in terms of $\mathrm{k}=2$ ) based on current techniques and technology. The "solar attenuator factor" refers to uncertainties related to the prelaunch characterization of the attenuator area and transmittance and on-orbit thermal effects and contamination causing knowledge of the transmittance and area to degrade with time. Figure 4 summarizes the best estimates for these errors based on commonly available laboratory facilities. The dominant error is due to the prelaunch characterization assuming periodic lunar views are available to assess on-orbit degradation. 
The earth-solar ratio term depends on the uncertainties in the earth view, solar view, and spectral effects. All of the factors in (4) can be categorized into one of these broad terms. The "spectral" term in the figure refers to uncertainties in the knowledge of the spectral characteristics of the spectrometers such as knowledge of the spectral band shape (wavelength calibration artifacts) or caused by the interaction of the spectrometer's slit with the imaging spectrometer (line spread function artifacts). Both terms can be characterized to the level of uncertainties shown in Figure 2.

The earth view and solar view are illustrated in Figures 4 and 5. The far left sets of boxes in both the solar and earth view are those uncertainties considered to be random in nature. The averaging approach of the CLARREO mission means that these uncertainties will be effectively zero for a direct benchmark and factors of 10 to 100 times less for the intercalibration, lunar, and solar view data sets due to the spatial and frame averaging that is planned. Assessment of SOLARIS data during laboratory testing with flight-like electronics will verify that averaging will indeed drive those errors to a level shown in the figures.

Sensor characteristics, boresight, solar integration, and electronics offset uncertainties represent a factor of two improvement over heritage approaches. CLARREOS's reliance on heritage hardware and designs, sufficient calibration and characterization time during the prelaunch phase, and thorough understanding of the instrument model will provide such improvements. The high-quality nature of the SIRCUS source permits better understanding of the inherent instrument effects permitting mitigation, correction, and characterization of the uncertainties. Larger values are expected for the earth view for sensor characteristics because of the complicated nature of the radiance source. The large range of expected radiances, the fact that the atmosphere and specular reflection create polarized states, and the spatial heterogeneity all create a situation that leads to greater earth view uncertainties than for the solar view.

Work by NIST with their laboratory version of SIRCUS has shown that stray light sensitivity of an imaging system can be determined to an order of magnitude better than methods widely used in other radiometric calibration facilities [4]. Efforts with SOLARIS have made the NIST laboratory SIRCUS closer to being a viable system for the flight version of the CLARREO RS sensor. Studies are still underway to develop the best approach for making a SIRCUS-like system available for CLARREO to ensure uncertainties are small enough to allow CLARREO to achieve the on-orbit requirements.

The electronics offset term would typically be one term ignored by most measurement programs. Scanning systems can readily determine these values on orbit. Even pushbroom systems that assess this effect at the start and end of an orbit can satisfactorily understand the variability of the offset at a level suitable for current applications. The unique accuracy requirements of CLARREO require system design that limits the variability in the offset as well as thorough characterization of the effect with temperature are necessary to ensure this term is negligible. Fortunately, such approaches are straightforward and do not represent a needed change in technology, simply careful application of known methods. 


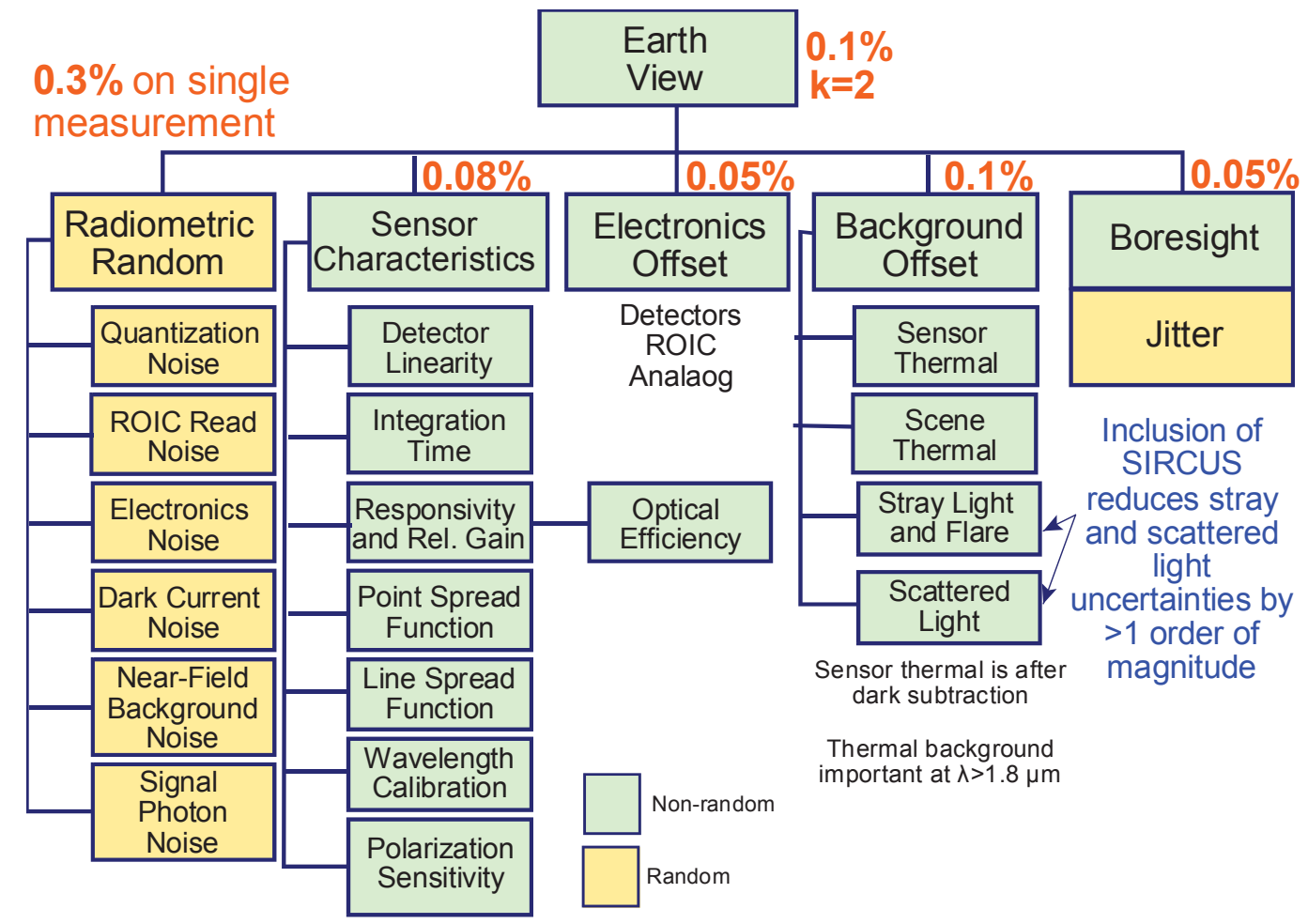

Figure 5 Error budget for the earth view measurement as estimated for RS sensor based on currently available technology.

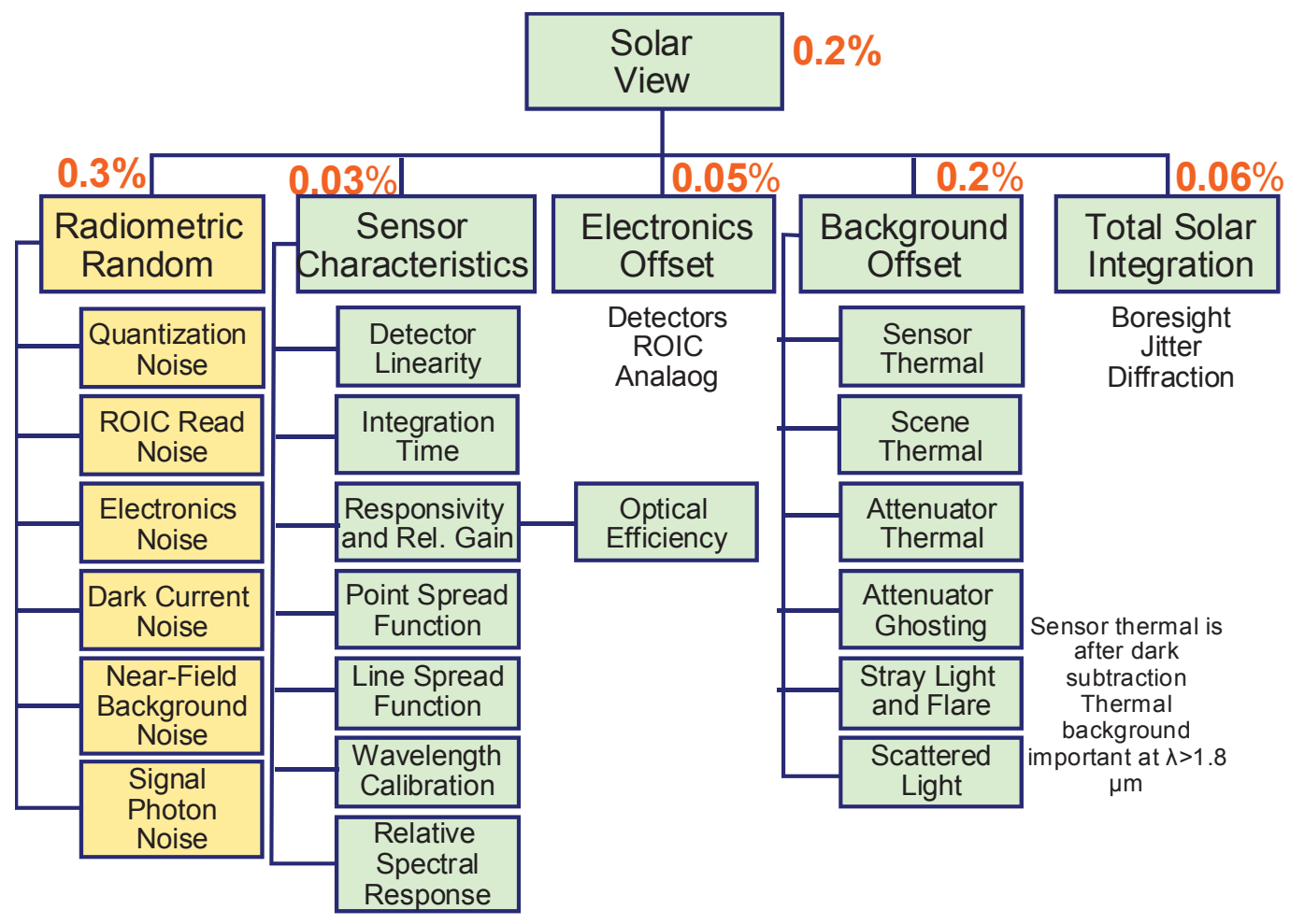

Figure 4 Error budget for the solar view measurement as estimated for RS sensor based on currently available technology 


\section{CONCLUSIONS}

The radiometric accuracy requirements needed to ensure that CLARREO RS can develop climate-quality benchmark data records necessitate improvements of current accuracies by nearly an order of magnitude. Incorporating a SIRCUSbased approach in collaboration with NIST provides the needed improvement in laboratory calibration to achieve the CLARREO accuracies. Demonstration of improvements is taking place through the SOLARIS CDS including evaluation of effects caused by the attenuator system as a function of time. SOLARIS gives the opportunity to include NIST-based laboratory calibrations both with monochromatic (SIRCUS) and broadband calibration approaches. SOLARIS testing will demonstrate "operational" use of SIRCUS while extending SIRCUS capabilities to wavelengths $>1$ micrometer. Key accomplishments with SOLARIS to date include:

1) Spectral response measurement using SIRCUS

2) Solar and lunar views

3) Reflectance retrievals relative to a field standard

Flat fielding and stability tests are still to be developed with all efforts leading to a physically-based spectrometer model including well-understood error budgets. Assessing polarization effects is still a challenge and SOLARIS will be used to demonstrate both the depolarizer technology of the sensor as well as the characterization of the polarization sensitivity of a CLARREO-like sensor.

Laboratory calibration protocols showing uncertainties at the levels given above are sufficient to the CLARREO mission and are to be reviewed by NIST and other metrological organizations. Included in the error budgets will be demonstration of the solar-view reflectance retrievals and an instrument model based transfer to orbit. Initial testing of SOLARIS has demonstrated the use of transfer radiometers for the calibration of an imaging spectrometer at accuracy levels seen in NIST laboratories

The end conclusion is that climate-quality accuracies are currently achievable at the monochromatic level for the calibration of laboratory radiometers. Implementing SIRCUS-like facilities outside of NIST coupled with careful sensor design to limit stray light and polarization sensitivity are critical to achieving the needed accuracy. In the end, adherence to these basic design and characterization principles will provide data at the accuracy required to provide the basis for a set of data records needed to understand the earth's climate.

\section{REFERENCES}

[1] Wielicki B. A., D. F. Young, M. G. Mlynczak, K. J. Thome, S. Leroy, J. Corliss, J. G. Anderson, C.O. Ao, R. Bantges, F. Best, K. Bowman, H. Brindley, J. J. Butler, W. Collins, J. A. Dykema, D. R. Doelling, D. R. Feldman, N. Fox, X. Huang, R. Holz, Y. Huang, Z. Jin, D. Jennings, D. G. Johnson, K. Jucks, S. Kato, D. B. Kirk-Davidoff, R. Knuteson, G. Kopp, D. P. Kratz, X. Liu, C. Lukashin, A. J. Mannucci, N. Phojanamongkolkij, P. Pilewskie, V. Ramaswamy, H. Revercomb, J. Rice, Y. Roberts, C. M. Roithmayr, F. Rose, S. Sandford, E. L. Shirley, W.L. Smith, Sr., B. Soden, P. W. Speth, W. Sun, P.C. Taylor, D. Tobin, X. Xiong, "Achieving Climate Change Absolute Accuracy in Orbit,". Bull. Amer. Met Soc., in press. doi: 10.1175/BAMS-D-12-00149.1.

[2] K. Thome, T. Gubbels, R. Barnes, " Preliminary error budget for the Reflected Solar Instrument for the Climate Absolute Radiance And Refractivity Observatory." Proc. SPIE Vol. 8153, San Diego, Calif., 2011.

[3] K. Thome, J. McCorkel, J. Hair, B. McAndrew, A. Daw, D. Jennings, and D. Rabin, " Test plan for a calibration demonstration system for the reflected solar instrument for the climate absolute radiance and refractivity observatory", Proc. SPIE Vol. 8516,.San Diego, Calif. 2012.

[4] Brown, S.W., Eppeldauer, G.P., and Lykke, K.R., "NIST facility for Spectral Irradiance and Radiance Responsivity Calibrations with Uniform Sources," Metrologia Vol. 37, 579-582, 2000. 